\title{
Análisis de las diferencias motivacionales entre el fútbol 7 y el fútbol 11
}

\section{Analysis of motivational differences between football 7 and 11}

\section{Análise das diferenças motivacionais entre o futebol 7 e o futebol 11}

\author{
R.C. Fernández Pérez', J.M. Yagüe Cabezón ${ }^{2}$, O. Molinero González ${ }^{3}$, S. Márquez Rosa y A. Salguero del Valle
}

1 IBOMED, 2 Universidad de León, 3 Universidad Autónoma de Chile

\begin{abstract}
Resumen: El objeto de este estudio es analizar las diferencias motivacionales que existen entre el fútbol 7 y el fútbol 11. Para ello trabajamos con una muestra de 183 futbolistas masculinos pertenecientes a tres clubes de fútbol base entre 8 y 18 ańos, a los cuáles se les administraron un Cuestionario Sociodemográfico, la Escala de Orientación Motivacional en el Deporte de Weiss, Bredemeier y Shewchuk, (1985), el Cuestionario de Orientación a la Tarea y al Ego de Duda y Nicholls, (1989) y la Escala de Habilidad Física Percibida de Ryckman, Robbins, Thornton y Cantrell (1982). Los participantes, tanto en fútbol 7 como en fútbol 11, mostraron una mayor orientación a la tarea que al ego, estaban más motivados intrínseca que extrínsecamente y poseían unos valores medio-altos en habilidad física percibida tanto general como específica. Los sujetos pertenecientes a la modalidad de fútbol 7 obtuvieron valores más elevados en orientación a la tarea $8,85( \pm 1,13)$, en motivación intrínseca $8,64( \pm 1,10)$ y en habilidad física percibida general $7,45( \pm 1,03)$ y específica $7,52( \pm 1,81)$, escalas que indican y aseguran la permanencia en la práctica, aunque también alcanzaron mayor puntuación que los practicantes de fútbol 11 en orientación al ego 5,34 $( \pm 2,32)$, motivación extrínseca 7,44 $( \pm 1,77)$ y amotivación $3,21( \pm 2,06)$, lo que podría atribuirse a que son deportistas con un bagaje de experiencias aún corto en el fútbol y con un conocimiento no demasiado elevado del deporte en sí.

Palabras clave: Fútbol, motivación, orientación de metas, habilidad física percibida.

Abstract: The purpose of our study is to analyze the motivational differences between soccer 7 and soccer 11. Participants were of 183 male players from three soccer clubs, aged 8 to 18 years. They were administered a Sociodemographic Questionnaire, the Motivational Orientation in Sport Scale by Weiss, Bredemeier y Shewchuk, (1985), the Task and Ego Orientation in Sport Questionnaire by Duda y Nicholls, (1989) and the Perceived Physical Ability Scale by Ryckman, Robbins, Thornton y Cantrell (1982). Both
\end{abstract}

soccer 7 and 11 participants scored higher in task than in ego orientation, were more intrinsicalley that extrinsically motivated and reached mediun to high values in both general and specific perceived physical ability. Soccer 7 participants reached higher scores than soccer 11 athletes in task orientation $8,85( \pm 1,13)$, intrinsic motivation $8,64( \pm 1,10)$ and perceived physical ability 7,45 $( \pm 1,03)$ and specific $7,52( \pm 1,81)$, scales related to sport adherence, though also scored higher in ego orientation $5,34( \pm 2,32)$, extrinsic motivation 7,44 $( \pm 1,77)$ and amotivation $3,21( \pm 2,06)$, which could be related to their low experience and knowledge of the practised sport. Key words: Soccer, motivation, goal orientation, perceived physical ability. Resumo: O objetivo deste estudo é analisar as diferenças motivacionais entre o futebol 7 e o futebol 11 . A amostra está composta por 183 jogadores do sexo masculino de três clubes de base entre 8 e 18 anos. Foram aplicados os seguintes instrumentos: um questionário sociodemográfico, Escala de Orientação Motivacional no Esporte Weiss, Bredemeier e Shewchuk (1985 ), Questionário de Orientação de Tarefas e Ego Duda e Nicholls (1989) e a Escala de Capacidade Física Percebida de Ryckman, Robbins, Thornton e Cantrell (1982). Os sujeitos da modalidade de futebol 7 obtiveram valores maiores na orientação a tarefa $(8,85 \pm 1,13)$, em motivação intrínseca $8,64( \pm 1,10)$ e capacidade física percebida geral 7,45 $( \pm 1,03)$ e específica $7,52( \pm 1,81)$, escalas que indicam e asseguram a permanência na prática, no entanto, também alcançaram pontuaçóes maiores que os praticantes de futebol 11 na orientaçâo ego $5,34( \pm 2,32)$, motivação extrínseca 7,44 ( \pm 1,77 ) e desmotivação 3,21 ( $\pm 2,06$ ), o que poderia ser atribuído a pouca bagagem de experiência dos atletas de futebol, assim como um limitado conhecimento do esporte em si.

Palavras-chave: futebol, motivação, orientação de metas, habilidades física percebida.

\section{Introducción}

Actualmente el fútbol constituye un fenómeno que ha adquirido una trascendencia vital en nuestra sociedad. Contribuye a la salud física, al equilibrio psíquico y al bienestar social de quienes lo realizan, y fomenta una serie de hábitos y valores que repercuten en una mayor integración del individuo en su entorno. Es por ello, que en la actualidad uno de los objetivos principales de los programas de formación es intentar man-

Dirección para correspondencia [Correspodence address]: Roberto Carlos Fernández Pérez. INCAFD (605) 047 023. Departamento de Educación Física y Deportiva e Instituto de Biomedicina (IBIOMED). Campus de Vegazana s/nº.C. P. 24007. Universidad de León. E-mail: roberlavirgen@ hotmail.com tener a los individuos en la práctica, evitando el abandono prematuro.

Sería conveniente profundizar en las características de los practicantes, con el objetivo de concretar cuáles son los motivos que le arrastran a practicar este deporte, lo que servirá de guía para establecer estrategias específicas que inciten a los sujetos a mantenerse en la práctica deportiva iniciada. Figueiredo, Gonzalves, Silva y Malina (2009), afirman que niveles elevados en habilidad física percibida aseguran la permanencia del sujeto en la práctica; Ommundsen, Roberts, Nicolas y Miller (2005), aseguran que valores elevados en habilidad física percibida y en motivación intrínseca predicen que el futbolista seguirá vinculado a la práctica; Junge, Rosch, Pe- 
terson, Baumann y Dvorak (2002) concluyen que niveles de habilidad física percibida altos aseguran la práctica en el tiempo y Ullrich-French y Smith (2009) confirman que los futbolistas que se muestran más motivados intrínsecamente seguirán vinculados a la práctica.

Estamos ante un deporte en el que aparecen continuamente problemas motrices, que además no están de ningún modo previstos puesto que siempre varían en su orden o frecuencia de aparición y en su complejidad (Grehaigne, 2001). El proceso de entrenamiento no sólo debe respetar estas señas de identidad, sino que además debe exaltarlas, puesto que únicamente así el rendimiento se verá reforzado. Esta circunstancia exige la necesidad de encontrar y aplicar programas de intervención que sean fieles a las necesidades que reclama cada disciplina físico-motriz (Abad, Gimenez, Robles y Castillo, 2013).

El estudio del proceso de iniciación deportiva corresponde a un periodo comprendido entre los seis-siete y los catorcequince ańos aproximadamente, si bien se pueden dar modificaciones dependiendo del desarrollo individual de la experiencia previa que haya tenido cada practicante. Es innegable que dentro de estas edades existen periodos más sensibles que otros para el aprendizaje deportivo. Dentro del fútbol, la fase de formación recibe el nombre de fútbol base. Este es el periodo que el nińo va adquiriendo las habilidades propias del deporte hasta dominarlas (Morcillo, 2004; Mesquita, Borges, Rosado y De Souza, 2011; Vickers, y Schoenstedt, 2011), desde el inicio de la actividad en el fútbol, hasta su acceso al proceso de máximo especialización (Sans y Frattarola, 2009; Giménez, Abad y Robles, 2010). En la actualidad, en la mayoría de comunidades, la categoría benjamín (8-9 años) y alevín (10-11 años), compiten en la modalidad de fútbol 7 , mientras que la categoría infantil (12-13 años), cadete (14-15 años) y juvenil (16-18 ańos) lo hacen en fútbol 11.

Todo ello hace vislumbrar un panorama dentro del fútbol base, marcado por una serie de parámetros que lo aleja de lo que es la esencia de la iniciación deportiva, que es la formación, con lo cual cada vez es más frecuente ver casos de abandono prematuro de la práctica deportiva debido a experiencias desagradables y en muchos de los casos evitables, de haber seguido unas pautas y una metodología de trabajo adaptada y a través de educadores con la formación necesaria como para afrontar procesos de enseńanza-aprendizaje en etapas de formación. En el caso concreto del fútbol, se atribuyen como principales causas para el abandono del mismo, otras cosas que hacer, falta de diversión, poco tiempo de juego y problemas con el entrenador, para Narciso, Otto y Mielke (1984); conflicto de intereses, excesivo énfasis en la competición y poca comunicación, para Pooley (1980). En cuanto a cifras de abandono de la práctica deportiva existen múltiples estudios como los realizados en 1978, por Seefeldt et al., recogidos por Weinberg y Gould (1996), manifiestan que entre los 13 y 18 años de edad el 80\% de los jóvenes han abandonado la práctica deportiva, con lo que se puede extraer que si bien es cierto que la participación en los programas deportivos aumenta, también el número de abandonos es numeroso, presentándose ésta última como una de las áreas de estudio de naturaleza psicológica de mayor importancia en el deporte infantil y juvenil (Gould, Feltz, Weiss y Petlichkoff, 1982; Da Cunha, Mesquita, Rosado, Sousa y Pereira, 2010).

Otros estudios, tasan el abandono alrededor del $35 \%$ en la edad juvenil, es decir, de cada 10 niños implicados 3-4 abandonan al llegar a los 14-15 años (Weinberg y Gould, 1996). Otros trabajos como los llevados a cabo por Medbery y Gould, (1998) y por Stratton, (1999) arrojan unos resultados un poco más optimistas y reducen este porcentaje hasta el $25 \%$ a nivel de práctica deportiva y al $30 \%$ en el caso concreto de la natación respectivamente.

Diversas teorías han tratado y profundizado en el estudio de la motivación. Teorías que abarcan desde posiciones mecanicistas, que conciben al ser humano como un sujeto a merced de las influencias del entorno, hasta las perspectivas que destacan el papel de la cognición en las respuestas de los sujetos. La idea fundamental de esta perspectiva es que las personas son organismos intencionales, dirigidos por sus objetivos y que actúan de forma racional de acuerdo con estos objetivos (Nicholls, 1984). Según Maehr y Nicholls (1980) el primer paso necesario para entender las conductas de logro, es reconocer que el éxito y el fracaso son estados psicológicos basados en el significado subjetivo que la persona le da al logro. El éxito, el fracaso y el logro, después del resultado en una acción, dependen de cómo una persona lo reconozca en función de su meta de logro, así lo que para unos es interpretado como éxito para otros es interpretado como fracaso.

Una de las teorías más relevantes en el estudio de la motivación en el deporte, que se engloba dentro de lo que se conoce como perspectiva interaccional, y que ha puesto una corriente muy fructífera de producción científica, es la Teoría de las Metas de Logro (Nicholls, 1989), que a continuación se desarrollará detenidamente. En 1978, Harter define un modelo motivacional llamado Percepción de Competencia, que en la década de los 80 y gracias a los trabajos en el ámbito educativo de Ames (1987), Ames y Archer (1987, 1988), Dweck y Elliott (1983), Dweck y Leggett (1988) y Maehr y Nicholls (1980), en los que se enuncia que los componentes direccionales de la motivación (objetivos) determinan en gran medida las conductas de logro que aparecen en un entorno competitivo.

Dentro de esta línea en la motivación se encuentra la Teoría de las Metas de Logro, con diferentes orientaciones: una que va a ir hacia la ejecución de la tarea, centrándose la persona en la realización del trabajo asignado; y la segunda, en la que la persona lo que busca es el resultado, ganar o quedar mejor que otro. Por otro lado, y atendiendo a la Teoría de 
la Autodeterminación, los dos aspectos de la motivación que más atención han recibido en el campo de la psicología del deporte y de la práctica deportiva han sido los constructos de motivación intrínseca y motivación extrínseca.

Los aspectos motivacionales comparados en este estudio son, en primer lugar la motivación, la cuál ha sido definida de diferentes maneras. La mayoría de autores coinciden en referirla a la intensidad y dirección del esfuerzo o del comportamiento (Sage y Loudermilk, 1979; Isorna, Rial y Vaquero, 2014). La dirección se refiere a sí el individuo busca, se aproxima o se siente atraído por ciertas situaciones y la intensidad se refiere a la cantidad de empeño que una persona emplea en una situación determinada (Weinberg y Gould, 1996; Granero y Baena, 2013). Las razones que conducen a los individuos a la práctica de una actividad deportiva pueden ser de distinta naturaleza, y se han clasificado como externas e internas (Escartí y Cervelló, 1994). Estos dos tipos de motivaciones no son excluyentes entre sí, y aunque la tendencia es que predomine una sobre la otra, se pueden dar juntas perfectamente en un mismo individuo (González, Sicilia y Moreno, 2011).

En segundo lugar, en el ámbito del deporte, diversos autores argumentan que para comprender el significado y las razones por las cuales los deportistas se implican y están motivados en los entrenamientos es necesario analizar, entre otros aspectos, las perspectivas de meta que adoptan en ese contexto de logro concreto (Roberts y Treasure, 1995; Duda, 1993). Dos son las perspectivas de meta que predominan y fueron denominadas por Nicholls (1984) como estado de implicación a la tarea y estado de implicación al ego. En general los sujetos que están orientados a la tarea mostrarán conductas, afectos y cogniciones que conllevarán la máxima motivación, independientemente del nivel de sus percepciones de habilidad (Moreno, Cervelló, Zomeño y Marín, 2009). Una orientación hacia la tarea se refleja en la percepción por parte del sujeto de que el éxito proviene del esfuerzo y de la mejora personal. Por otro lado los sujetos orientados al ego se caracterizarán por un modelo adaptativo de logro si muestran percepciones de habilidad consistentemente altas; en este caso estarán motivados a persistir y demostrar a otros su competencia. Una orientación hacia el ego supone que la percepción de éxito se basa en superar a los demás, y los su- jetos demuestran si son o no son competentes comparándose con otros (Duda, 1993).

Por último, la habilidad física percibida hace referencia a las expectativas sobre la propia capacidad de alcanzar un determinado nivel de ejecución, que es una parte del proceso conductual del deportista, cuyas consecuencias producirán un determinado resultado. Por este motivo debemos hacer énfasis en centrar la atención selectiva durante la competición en las variables del proceso y no del resultado, ya que a menudo se interpretan erróneamente los conceptos de "expectativas del resultado" y "expectativas de eficacia”, lo cual va a generar confusión y que generalmente irá acompañado de comportamientos ineficaces (Bandura, 1986). Cuando hablamos de habilidad física percibida no nos estamos refiriendo al nivel de habilidad real que tiene un individuo, sino a la creencia personal de ese sujeto, que podrán encontrarse más o menos cercanas. La auto-eficacia, entonces, puede ser considerada una situación de auto-confianza específica (Feltz, 1988).

El propósito de este estudio es analizar las diferencias motivacionales de los futbolistas de la modalidad de fútbol 7 , de ocho a once años comprendiendo las categorías benjamín y alevín, y la de los futbolistas de fútbol 11, de doce a dieciocho años en categoría infantil, cadete y juvenil, basándonos en el análisis de la motivación, la orientación de metas y la habilidad física percibida. Partimos de la hipótesis de que los futbolistas pertenecientes a la modalidad de fútbol 7 presentan unos valores más elevados en orientación de metas, motivación y habilidad física percibida que los futbolistas de fútbol 11. Por el contrario, creemos que son los futbolistas de la modalidad de fútbol 11 los que en relación a la orientación de metas, están más orientados al ego.

\section{Método}

\section{Participantes}

La muestra de nuestro estudio estuvo compuesta por 183 futbolistas masculinos con edades comprendidas entre los $8 \mathrm{y}$ 18 años, con una media de $16,6( \pm 2,61)$ años, pertenecientes a tres clubes de fútbol base, ocupando así toda la franja del fútbol formativo, como podemos observar en la tabla 1 . 
Tabla 1. Distribución de los programas de entrenamiento por los diferentes equipos y categorías participantes en el estudio.

\begin{tabular}{|c|c|c|c|c|}
\hline $\begin{array}{c}\text { EQUIPOS } \\
\text { PARTICIPANTES }\end{array}$ & JUGADORES & $\begin{array}{c}\text { PROGRAMA } \\
\text { METODOLÓGICO }\end{array}$ & $\begin{array}{c}\text { No } \\
\text { SESIÓN }\end{array}$ & $\begin{array}{c}\text { No TOTAL } \\
\text { POR CATEGORÍA }\end{array}$ \\
\hline $\begin{array}{l}\text { C. D. Virgen del Camino } \\
\text { Benjamín A }\end{array}$ & 12 & Global & 12 & \multirow{3}{*}{38 Benjamines } \\
\hline $\begin{array}{l}\text { C. D. Virgen del Camino } \\
\text { Benjamín B } \\
\end{array}$ & 12 & Analítico & 12 & \\
\hline $\begin{array}{l}\text { C. D. Huracán Z } \\
\text { Benjamín }\end{array}$ & 14 & GR. CONTROL & 12 & \\
\hline $\begin{array}{c}\text { C. D. Virgen del Camino } \\
\text { Alevín A } \\
\end{array}$ & 12 & Global & 12 & \multirow{3}{*}{37 Alevines } \\
\hline $\begin{array}{c}\text { C. D. Virgen del Camino } \\
\text { Alevín B } \\
\end{array}$ & 12 & Analítico & 12 & \\
\hline $\begin{array}{c}\text { C. D. Huracán Z } \\
\text { Alevín }\end{array}$ & 13 & GR. CONTROL & 12 & \\
\hline $\begin{array}{c}\text { C. D. Virgen del Camino } \\
\text { Infantil } \\
\end{array}$ & 16 & Analítico & 12 & \multirow{3}{*}{49 Infantiles } \\
\hline $\begin{array}{c}\text { C. D. Ejido } \\
\text { Infantil } \\
\end{array}$ & 18 & Global & 12 & \\
\hline $\begin{array}{l}\text { C. D. Huracán Z } \\
\text { Infantil }\end{array}$ & 15 & GR. CONTROL & 12 & \\
\hline $\begin{array}{c}\text { C. D. Virgen del Camino } \\
\text { Cadete }\end{array}$ & 18 & Analítico & 12 & \multirow{3}{*}{52 Cadetes } \\
\hline $\begin{array}{l}\text { C. D. Ejido } \\
\text { Cadete }\end{array}$ & 16 & Global & 12 & \\
\hline $\begin{array}{c}\text { C. D. Huracán Z } \\
\text { Cadete }\end{array}$ & 18 & GR. CONTROL & 12 & \\
\hline $\begin{array}{c}\text { C. D. Virgen del Camino } \\
\text { Juvenil }\end{array}$ & 16 & Analítico & 12 & \multirow{3}{*}{50 Juveniles } \\
\hline $\begin{array}{l}\text { C. D. Ejido } \\
\text { Juvenil }\end{array}$ & 18 & Global & 12 & \\
\hline $\begin{array}{l}\text { C. D. Huracán Z } \\
\text { Juvenil }\end{array}$ & 16 & GR. CONTROL & 12 & \\
\hline
\end{tabular}

\section{Instrumentos}

Para recoger la información y poder alcanzar los objetivos establecidos, utilizamos cuatro cuestionarios. Para la evaluación de las características socio-demográficas se ha utilizado un cuestionario elaborado por el equipo encargado de la investigación compuesto por 17 items. Para determinar el grado de motivación de los sujetos, administramos la versión espańola de la Escala de Orientación Motivacional en el Deporte de Weiss, Bredemeier y Shewchuk, (1985), de Núñez, MartínAlbo, Navarro y González (2006), con las sub-escalas motivación intrínseca, extrínseca y amotivación, con 28 items. Para medir las orientaciones de meta utilizamos la versión española del Cuestionario de Orientación a la Tarea y al Ego, de Duda y Nicholls, (1989), de Balaguer, Castillo y Tomás (1996), con 6 ítems para la sub-escala ego y 7 ítems para la sub-escala tarea. Para valorar la habilidad física percibida administramos la versión española de la Escala de Habilidad Fisica Percibida de Ryckman, Robbins, Thornton y Cantrell (1982), con dos sub-escalas, una que recogía los 10 ítems originales de la escala para medir la habilidad física percibida general, y otra con 6 ítems para medir la Habilidad Física Percibida Específica, los cuales recogen de forma genérica los distintos gestos técnico-tácticos que engloban el deporte objeto de este trabajo, basándonos en los estudios de Salguero et al. (2003) y anteriormente de McAuley y Gill (1983).

\section{Procedimiento}

La administración de los cuestionarios se efectuó dentro del periodo de competición, en la primera sesión de entrenamiento de la semana, en los vestuarios que habitualmente se 
cambiaban, ya que reunían las condiciones espaciales y de comodidad necesarias, y en el horario de entrenamiento marcado en sus respectivos clubes. Antes de dicha administración, se explicó detalladamente a los participantes en qué consistía el estudio, cuál era su finalidad, y para qué se requería su participación. Asimismo, fueron descritos los cuestionarios, el orden en el que iban a ser administrados y que datos se intentaban conseguir en cada uno de ellos. El tiempo máximo concebido para cumplimentar los cuestionarios fue de $45 \mathrm{mi}$ nutos. De igual modo todos los participantes firmaron una hoja de consentimiento así como una autorización paterna para los menores de edad.

\section{Análisis de datos}

Se llevó a cabo una estadística descriptiva con el fin de hallar la media aritmética y la desviación típica en función de las variables estudiadas. Seguidamente, un análisis de varianza de una vía (ANOVA), para determinar la existencia o no de diferencias significativas entre grupos para los factores motivación, orientación de metas y habilidad física percibi$\mathrm{da}$, en función de la variable independiente (categoría competitiva), teniendo en cuenta un nivel de significación de $\mathrm{p} \leq 0,05$. Por último, y para analizar la relación entre factores y comprobar la existencia de diferencias significativas, se ha utilizado un análisis de correlación de Pearson. En este procedimiento estadístico, se aceptaron los resultados con grados de significación $\mathrm{p} \leq 0,05$. Todo el proceso estadístico fue realizado utilizando el paquete estadístico IBM SPSS Statistics, Versión 19 para Windows.

\section{Resultados}

Tras el análisis de los resultados obtenidos, pudimos observar como los futbolistas de nuestra muestra pertenecientes a la modalidad de fútbol 7 se mostraron más orientados a la tarea que al ego al igual que en fútbol 11. Tanto para la orientación a la tarea como para la orientación al ego las diferencias son significativas entre ambas categorías (Tabla 2).

Tabla 2. Puntuaciones en las subescalas del Cuestionario de Orientación a la Tarea y al Ego.

\begin{tabular}{|c|c|c|c|c|c|}
\hline ESCALA & CATEGORÍA & $\mathrm{N}$ & MEDIA & $\mathrm{SD}$ & $\mathrm{p}$ \\
\hline \multirow{2}{*}{ Orientación al ego } & Fútbol 7 & 59 & 5,34 & 2,32 & \multirow{2}{*}{$0,03^{*}$} \\
\hline & Fútbol 11 & 124 & 4,57 & 2,17 & \\
\hline \multirow{2}{*}{ Orientación a la tarea } & Fútbol 7 & 59 & 8,85 & 1,13 & \multirow{2}{*}{$0,001^{* *}$} \\
\hline & Fútbol 11 & 124 & 7,96 & 1,32 & \\
\hline
\end{tabular}

${ }^{*} \mathrm{p} \leq 0,05,{ }^{* *} \mathrm{p} \leq 0,01$.

Respecto a la motivación intrínseca y extrínseca, los participantes, tanto en la modalidad de fútbol 7 como en fútbol 11, resultaron estar más motivados intrínseca que extrínseca- mente. Las puntuaciones alcanzadas en motivación intrínseca, así como en motivación extrínseca diferían significativamente entre ambas categorías, no así la amotivación (Tabla 3).

Tabla 3. Puntuaciones en las subescalas de la Escala de Orientación Motivacional en el Deporte.

\begin{tabular}{|c|c|c|c|c|c|}
\hline ESCALA & CATEGORÍA & $\mathrm{N}$ & MEDIA & $\mathrm{SD}$ & $\mathrm{p}$ \\
\hline \multirow{2}{*}{ Motivación intrínseca } & Fútbol 7 & 59 & 8,64 & 1,10 & \multirow{2}{*}{$0,001^{* *}$} \\
\hline & Fútbol 11 & 124 & 7,63 & 1,38 & \\
\hline \multirow{2}{*}{ Motivación extrínseca } & Fútbol 7 & 59 & 7,44 & 1,77 & \multirow{2}{*}{$0,001^{* *}$} \\
\hline & Fútbol 11 & 124 & 6,08 & 1,59 & \\
\hline \multirow{2}{*}{ Amotivación } & Fútbol 7 & 59 & 3,21 & 2,06 & \multirow{2}{*}{ n.s. } \\
\hline & Fútbol 11 & 124 & 2,85 & 1,83 & \\
\hline
\end{tabular}

${ }^{*} \mathrm{p} \leq 0,05,{ }^{* *} \mathrm{p} \leq 0,01$. n.s. no significación

Por último, en lo que se refiere a la habilidad fisica percibida, se puso de manifiesto la existencia de diferencias significati- vas entre ambas categorías competitivas en habilidad física general, pero no en habilidad física percibida especifica (Tabla 4). 
Tabla 4. Puntuaciones en las subescalas de la Escala de Habilidad Física Percibida. ${ }^{*} \mathrm{p} \leq 0,05,{ }^{* *} \mathrm{p} \leq 0,01$.

\begin{tabular}{|c|c|c|c|c|c|}
\hline ESCALA & CATEGORÍA & $\mathrm{N}$ & MEDIA & $\mathrm{SD}$ & $\mathrm{p}$ \\
\hline \multirow{2}{*}{ Habilidad física percibida general } & Fútbol 7 & 59 & 7,45 & 1,03 & \multirow{2}{*}{$0,001^{* *}$} \\
\hline & Fútbol 11 & 124 & 6,66 & 1,11 & \\
\hline \multirow{2}{*}{ Habilidad física percibida específica } & Fútbol 7 & 59 & 7,52 & 1,81 & \multirow{2}{*}{ ns } \\
\hline & Fútbol 11 & 124 & 7,07 & 1,47 & \\
\hline
\end{tabular}

En la Figura 1 podemos observar a modo de resumen los resultados obtenidos por los futbolistas de nuestra muestra en las diferentes escalas en las modalidades de fútbol, 7 y fútbol
11. Cabe resaltar que los futbolistas de la modalidad de fútbol 7 puntúan más alto en todas las escalas que los futbolistas de fútbol 11.

Figura 1. Puntuaciones obtenidas en Fútbol 7 y 11 en todas las escalas. ${ }^{*} \mathrm{p} \leq 0,05,{ }^{* * *} \mathrm{p}=0,000$.

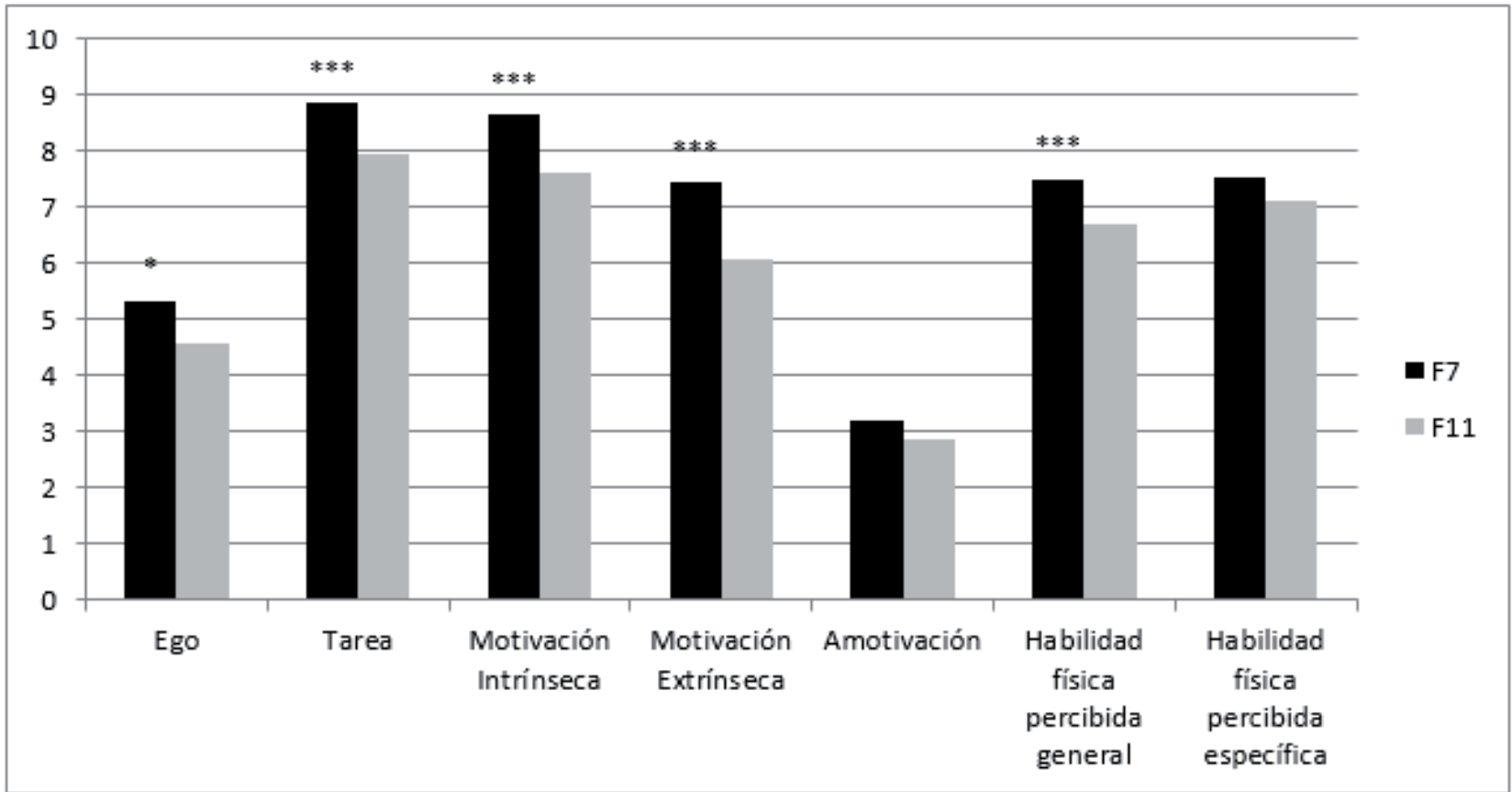

En cuanto al análisis correlacional entre las diferentes escalas, como podemos observar en la Tabla 5, la orientación al ego se correlaciona positivamente con la habilidad física percibida general, con la habilidad física percibida especifica, con la motivación extrinseca y con la amotivación. La orientación a la tarea correlaciona de forma positiva con la habilidad física percibida general, con la habilidad física percibida especifica, con la motivación intrinseca y con la motivación extrínseca, y negativamente con la amotivación. La motivación intrínseca se correlaciona de forma positiva con la motivación extrinseca. La habilidad física percibida general se correlaciona positivamente con la habilidad física percibida especifica, con la motivación intrínseca y con la motivación extrínseca. Y por último la habilidad física percibida especifica se correlaciona de manera positiva con la motivación intrínseca y con la motivación extrínseca. 
Tabla 5. Análisis correlacional entre las diferentes escalas. ${ }^{*} \mathrm{p} \leq 0,05,{ }^{* *} \mathrm{p} \leq 0,01$.

\begin{tabular}{ccccccc}
\hline & Tarea & HFP general & HFP específica & Motivación intrínseca & Motivación extrínseca & Amotivación \\
\hline Ego &, 061 &, $350^{* *}$ &, $298^{* *}$ &, 110 &, $411^{* *}$ &, $331^{* *}$ \\
Sig.(2-tailed) &, 485 &, 000 &, 000 &, 207 &, 000 &, 000 \\
\hline Tarea & &, $509^{* *}$ &, $373^{* *}$ &, $786^{* *}$ &, $273^{* *}$ & $-201^{*}$ \\
Sig.(2-tailed) &, 000 &, 000 &, 000 &, 001 & 0,20 \\
\hline HFP general & &, $601^{* *}$ &, $603^{* *}$ &, $491^{* *}$ &, 070 \\
Sig.(2-tailed) & &, 000 &, 000 &, 000 &, 419 \\
\hline HFP específica & &, $403^{* *}$ &, $354^{* *}$ &, 069 \\
Sig.(2-tailed) & & &, 000 &, 427 \\
\hline Motivación intrínseca & & & &, $469^{* *}$ &,- 130 \\
Sig.(2-tailed) & & &, 000 &, 134 \\
\hline Motivación extrínseca & & & & &, $409^{* *}$ \\
Sig.(2-tailed) & & & & & \\
\hline
\end{tabular}

\section{Discusión}

En nuestros estudio encontramos puntuaciones más elevadas en tarea que en ego, al igual que se ha detectado en investigaciones como las de Duda et al. (1995); Duda (1999); Cervelló, Escartí y Balagué (1999); Carlin, Salguero, Márquez y Garcés (2009). Camargo, Hirota y Verardi (2008) para futbolistas femeninas brasileñas, al igual que Hirota y De Marco (2006), y Figueiredo, Gonçalves, Cohelo e Silva y Malina (2009) con futbolistas obtuvieron del mismo modo que nuestro estudio, que eran los deportistas de categorías inferiores los que mayor orientación al ego tenían debido a que querían parecerse a sus ídolos en el deporte profesional.

Al igual que en nuestro estudio, Castillo, Duda, Álvarez, Mercé y Balaguer (2011) encontraron que los futbolistas cadetes se orientaron más a la tarea que al ego, valorando más el esfuerzo personal que la comparación y rivalidad interpersonal. Adie, Duda y Ntoumanis (2010) obtuvieron una correlación positiva entre la orientación a la tarea y predictores de continuidad en la práctica del fútbol, debido a que a partir de esta categoría los deportistas tienen claro que va a ser el esfuerzo, la continuidad y la capacidad de superación las que les hagan conseguir sus objetivos. Potgieter y Steyn (2010) en jóvenes futbolistas comprobaron como la orientación a la tarea se correlacionó más positivamente que el ego hacia el éxito y el fracaso. Cetinkalp y Turksoy (2011) observaron con jóvenes futbolistas como se orientaban más hacia la tarea, relacionándose con el desarrollo de habilidades y el sentimiento de afinidad y continuidad en la práctica, ya que es a través del esfuerzo y la continuidad el camino para poder llegar a conseguir sus objetivos.

En cuanto a la motivación los futbolistas de nuestra muestra se encontraron más motivados intrínseca que extrínsecamente en la misma línea que los estudios de Fry, McCle- ments y Sefton (1981) en el hockey sobre hielo; Balaguer y Atienza (1994) y Villamarín, Mauri y Sanz (1998) en el tenis; Kirkby, Kolt y Liu (1999) y Ortega y Zubiaur (2003) en la gimnasia; González, Tabernero y Márquez (2000), en jóvenes tenistas y futbolistas; Salguero, González-Boto, Tuero y Márquez (2004); Andrade, Salguero y Márquez (2006) y Andrade, Salguero, González-Boto y Márquez (2008) todos ellos en nadadores; Andrade, Salguero, González y Márquez (2006) en deportistas adultos; Martínez, Bastos y Salguero (2005) y Martínez el al. (2008) en el fútbol. Ullrich-French y Smith (2009), también con jóvenes futbolistas, observaron que estaban más motivados intrínseca que extrínsecamente. Todos ellos, al igual que en nuestra muestra, tenían motivos inherentes a la práctica para seguir realizando deporte, ya que son los principios y los valores que les van a llevar a conseguir realizarse dentro de su práctica deportiva. Caglar y Hülya (2010), a diferencia de nuestro estudio concluyeron que a mayor categoría competitiva mayor motivación extrínseca y mayor nivel de habilidad física percibida en deportes de equipo incluido el fútbol, ya que según su estudio la cercanía a la etapa profesional les hacía estar más centrados en las posibles recompensas que pudieran obtener de ese paso. Wood y Wilson (2012) obtuvieron valores semejantes a los nuestros en habilidad física percibida en el lanzamiento de penalti en fútbol. Morano, Colella y Capranica (2011) corroboran como los deportistas de diferentes deportes de equipo incluido el futbol, consiguieron unos valores en habilidad física percibida mayores que los deportistas de deportes individuales. Price y Weiss (2011) con jóvenes futbolistas obtienen al igual que en nuestro estudio un alto nivel de habilidad física percibida, relacionándolo con una buena capacidad de liderazgo. Los futbolistas de la modalidad de fútbol 7 son lo que obtienen puntuaciones mayores en habilidad física percibida específica, lo cuál puede ser debido a que los jugadores más jóvenes al 
tener menos experiencia en el deporte piensan que ya lo dominan perfectamente.

Los valores más elevados de habilidad física percibida corresponden a la categoría de menor edad, es decir al fútbol 7 , al igual que los resultados de Weigand y Broadhurst (1998) en fútbolistas; Salguero et al. (2004) y Andrade, Salguero, González-Boto y Márquez (2005) en nadadores. Es posible que a medida que la edad de los deportistas avanza, tengan un conocimiento más certero y preciso acerca de la ejecución de sus habilidades, ya que en el caso de los más pequeños al carecer de dicha experiencia cada tarea es novedosa, ayudándoles a mejorar cada día su capacidad y desarrollo de destrezas. En fútbol 11 se da una habilidad física percibida, tanto general como específica, menor que en fútbol 7; estando de igual modo menos orientados a la tarea y a la motivación intrínseca que en fútbol 7. En esta misma línea de resultados, a pesar de no ser nuestra muestra potencialmente predispuesta al abandono, se encuentran los estudios de Roberts (1995) que estimaron que el $80 \%$ de los adolescentes abandonan los programas deportivos organizados a todos los niveles entre los 12 y los 17 años por diferentes motivos como cambios de interés, incompatibilidad, problema con los compañeros y entrenadores, etc.. Así, Carlin, Salguero, Márquez y de los Fayos (2009) y Salguero, González-Boto, Tuero y Márquez (2003), citan en sus estudios como edad de abandono del deporte la edad de 18 años.

En cuanto a las correlaciones, observamos como la orientación al ego correlacionaba positivamente con la motivación extrínseca y amotivación, en la misma línea que Swain y Harwood (1996) que afirman que las percepciones sociales o externas son los mayores predictores de orientación al ego, estando relacionados con la necesidad de ganar, lo que se debe al interés en ser los mejores para conseguir recompensas y reconocimientos externos ajenos a la propia práctica deportiva. La orientación a la tarea correlacionó positivamente con la habilidad física percibida, general y específica, y con la motivación intrínseca, corroborado por varios autores (Duda, 1989; White y Duda, 1994; Castillo et al., 2000), quienes afirman que los deportistas orientados a la tarea buscan mejorar su nivel de habilidad y cooperar con los demás, y valoran la dimensión social de la experiencia deportiva. Podemos afirmar, basándonos en lo expuesto por Nicholls (1989), que el tipo de orientación de un deportista en situaciones de logro no solo va a depender de sus creencias acerca de las causas de éxito, sino que estará condicionado de igual modo por aspectos como la conducta, el esfuerzo o la cooperación.

La habilidad física percibida general correlacionó positivamente con la habilidad física percibida específica y la motivación intrínseca, y éstas dos entre ellas. Partiendo de los resultados obtenidos por Salguero (2004) podemos afirmar que los sujetos más motivados se perciben más hábiles, lo que ayudará a que puedan conseguir mejores resultados. Los da- tos de Feltz y Petlichkoff (1983), para los cuáles los sujetos con una percepción más baja de habilidad son más propensos al abandono, apoyan esta hipótesis. Otros estudios como los de Roberts et al. (1981), Klint y Weiss (1986), Kavussanu y Roberts (1996), Lázaro, Villamarín y Limonero (1996), Weigand y Broadhurst (1998) y Newton y Duda (1999) señalan resultados en este mismo sentido. Álvarez, Balaguer, Castillo y Duda (2012), en jóvenes futbolistas encontraron mayor motivación intrínseca y una correlación positiva de ésta con la intención de continuar en la práctica al igual que hemos encontrado en nuestro estudio. A diferencia de nuestra investigación, Leo et al. (2012) en su estudio con jóvenes futbolistas obtuvieron correlaciones entre una baja habilidad física percibida y nulas expectativas de llegar a la élite y viceversa, debido a que la baja percepción que tenían de su habilidad les hacía abandonar la práctica o cambiarse de disciplina.

\section{Conclusiones}

Los futbolistas participantes en nuestro estudio, tanto en la modalidad de fútbol 7 como en la de fútbol 11, presentan unos valores alejados de ser una muestra potencialmente predispuesta al abandono prematuro de la práctica del fútbol, ya que todos ellos muestran mayor orientación a la tarea que al ego, están más motivadas intrínseca que extrínsecamente y poseen unos valores medio-altos en habilidad física percibida tanto general como específica. Los instrumentos utilizados son una buena herramienta para predecir que sujetos podrían estar potencialmente predispuestos a abandonar la práctica del fútbol de forma prematura.

En cuanto a las diferencias motivacionales entre ambas modalidades podemos concluir y afirmar la hipótesis de partida, que los futbolistas de nuestra muestra pertenecientes a la modalidad de fútbol 7 , obtuvieron valores más elevados en orientación a la tarea, en motivación intrínseca y en habilidad física percibida, tanto general como específica, escalas que indican y aseguran la permanencia en la práctica, aunque también obtuvieron mayor puntuación que los futbolistas de fútbol 11, en orientación al ego, aspecto que hace que neguemos esta hipótesis inicial, motivación extrínseca y amotivación, aspecto que podría atribuirse a que son futbolistas con un bagaje de experiencias aún corto en el fútbol y con un conocimiento no demasiado elevado del deporte en sí.

En base a nuestra experiencia profesional y con el objetivo de conseguir mantener estos valores motivacionales o incluso de mejorarlos, sería conveniente que en el fútbol base se impartieran programas de formación caracterizados por: climas motivacionales agradables que favorezcan los aprendizajes, tareas adaptadas al nivel madurativo y evolutivo del niño, progresión en la dificultad de las situaciones de enseńanza-aprendizaje y variedad de tareas y contenidos específicos, siempre a través de formas jugadas cercanas a la esencia 
del juego. Así mismo, se debería utilizar las correcciones y los refuerzos cuando la situación lo requiera y llevar a cabo una formación en valores que favorezca el esfuerzo, la constancia y el respeto tanto por los compańeros como los adversarios, de igual modo afrontar la competición como una situación más de aprendizaje y no como un fin en sí mismo respetando tanto al entrenador como al árbitro y adversarios.

Respecto a las limitaciones del estudio, sería interesante realizar el estudio de forma longitudinal con los mismos futbolistas, y observar las variaciones en los resultados en su paso del fútbol 7 a fútbol 11.

\section{Aplicaciones prácticas}

Atendiendo a la fundamentación teórica del trabajo y a los resultados obtenidos podemos señalar que la habilidad físi- ca percibida, los distintos tipos de motivación (intrínsecaextrínseca) y la orientación de metas (tarea-ego), podrían ser unos indicadores fiables del abandono deportivo, en este caso el fútbol, de manera que podremos prevenir o intervenir antes de que se produzca el mismo.

Se han detectado resultados positivos a nivel motivacional en la etapa o en el momento en el que tiene lugar en el cambio de fútbol 7 a futbol 11. Pensamos que estos niveles adecuados de motivación pueden generar una predisposición al aprendizaje de nuevas habilidades que debe ser aprovechado. No debemos olvidar que esta etapa tiene lugar una de esas fases sensibles y/o periodos críticos fundamentales de cara al aprendizaje deportivo, y su correcta planificación será fundamental para el rendimiento futuro de los jóvenes deportistas.

\section{Referencias Bibliográficas}

1. Abad, M. T., Gimenez, F. J., Robles, J. y Castillo, E. (2013). La formación de los entrenadores de jóvenes futbolistas. Revista de Ciencias del Deporte, 9, (2), 105-114.

2. Adie, J. W., Duda, J. L. y Ntoumanis, N. (2010). Achievement goals, competition appraisals, and the well- and ill-being of elite youth soccer players over two competitive seasons. Journal of Sport \& Exercise Psychology,32, 555-579.

3. Álvarez, M. S., Balaguer, I., Castillo, I. y Duda, J. L. (2012). The coachcreated motivational climate, young athletes'weil-being, and intentions to continue participation. Journal of Clinical Sport Psycttotogy, 6, 166-179

4. Ames, C. (1987). The enhancement of student motivation. En M. I. Maehr y D. A. Kleiber (Eds.), Vol. 5. Advances in motivation and achievement: Enhancing motivation (pp. 123-148). Greenwich, CT: JAI Press.

5. Ames, C. y Archer, J. (1987). Mother's beliefs about the role of ability and effort in school learning. Journal of Educational Psychology, 79(4), 409-414.

6. Ames, C. y Archer, J. (1988). Achievement goals in the classroom: Students' learning strategies and motivation processes. Journal of Educational Psychology, 80(3), 260-267.

7. Andrade, A., Salguero, A., González-Boto, R. y Márquez, S. (2005). Habilidad física percibida en nadadores de competición brasileńos. Cuadernos de Psicología del Deporte, 5, 245-255.

8. Andrade, A., Salguero, A., González-Boto, R. y Márquez, S. (2006). Motives for participation in physical activity by brazilian adults. Perceptual and Motor Skills, 1025, 358-367.

9. Andrade, A., Salguero, A., González-Boto, R. y Márquez, S. (2008). The relationship of participation motivation to goal orientations and perceived physical ability in brazilian swimmers. Psychologia. An International Journal of Psychological Sciences, 51 (3), 157-169.

10. Andrade, A., Salguero, A. y Márquez, S. (2006). Motivos para a participacao esportiva em nadadores brasileiros. Fitness \& Performance Journal, 5 (6), 363-369.

11. Balaguer, I. y Atienza, F. (1994). Principales motivos de los jóvenes para jugar al tenis. Apunts Educación Física y Deportes, 31, 285-299.

12. Bandura, A. (1986). Pensamiento y acción. Barcelona: Martínez Roca.

13. Carlin, M., Salguero, A., Márquez, S. y Garcés de Los Fayos, E. J. (2009). Análisis de los motivos de retirada de la práctica deportiva y su relación con la orientación motivacional en deportistas universitarios. Cuadernos de Psicología del Deporte, 9 (1), 85-99.

14. Caglar, E. y Hülya, F. (2010). Motivational cluster profiles of adoles- cent athletes: an examination of differences in physical-self perception. Journal of Sports Science and Medicine 9, 231-238.

15. Camargo, F., Hirota, V. B. y Verardi, C. E. (2008). Orientaçao motivacional na aprendizagem esportiva do futsal na escola. Revista Mackenzie de Educaçao Física e Esporte, 7 (3), 53-62.

16. Carlin, M., Salguero, A., Márquez, S. y Garcés, E. (2009). Estudio de las orientaciones de meta en deportistas universitarios: análisis de género, tipo de deporte y nivel competitivo. Revista Iberoamericana de Psicología del Ejercicio y del Deporte, 4 (2), 201-216.

17. Castillo, I., Balaguer, I. y Duda, J. (2000). Las orientaciones de meta y los motivos de práctica deportiva en los jóvenes deportistas valencianos escolarizados. Revista de Psicología del Deporte, 9, 37-50.

18. Castillo, I., Duda, J. L., Álvarez, M. S., Mercé, J. y Balaguer, I. (2011). Clima motivacional, metas de logro de aproximación y evitación y bienestar en futbolistas cadetes. Revista de Psicología del Deporte, 20 (1), 149-164.

19. Cetinkalp, Z. K. y Turksoy, A. (2011). Goal orientation and self-efficacy as predictors of male adolescent soccer player's motivation to participate. Social Behavior and Personality, 39 (7), 925-934.

20. Cervelló, E. M., Escartí, A. y Balagué, G. (1999). Relaciones entre la orientación de metas disposicional y la satisfacción de los resultados deportivos, las creencias sobre las causas de éxito en el deporte y la diversión con la práctica deportiva. Revista de Psicología del deporte, 8 (1), 7-19.

21. Da Cunha, G., Mesquita, I. M., Rosado, A. F., Sousa, T., e Pereira, P. (2010). Necessidades de formaçao para o exercício profissional na perspectiva do treinador de futebol em funçao da sua experiencia e nível de formaçao. Motriz. Revista de Educaçao Física, 16(4), 931-941.

22. Duda, J. L. (1989). Relationship between task and ego orientation and the perceived purpose of sport among high school athletes. Journal of Sport and Exercise Psychology, 11, 318-335.

23. Duda, J. L. (1993). A social cognitive approach to the study of motivation in sport. En R. N. Singer, M. Murphey y M. Tennant (Eds). Handbook of research on sport psychology. New York: McMillan.

24. Duda, J. L. (1999). El clima motivacional y sus implicaciones para la motivación, la salud y el desarrollo de los desórdenes de alimentación en gimnastas. Revista de Psicología Social Aplicada, 9 (1), 7-23.

25. Duda, J. L., Chi, L., Newton, M. L., Walling, M. D. y Catley, D. (1995). Task and ego orientation and intrinsic motivation in sport. International journal of sport psychology, 26 (1), 40-63. 
26. Dweck, C. S. y Elliott, E. S. (1983). Achievement motivation. En E. M. Hetherington (Ed.), Handbook of child psychology: Socialization, personality, and social development (4rd ed., pp. 643-691). New York, NY: John Wiley \& Sons.

27. Dweck, C. S. y Leggett, E. L. (1988). A social-cognitive approach to motivation and personality. Psychological Review, 95(2), 256-273.

28. Escartí, A. y Cervelló, E. (1994). La motivación en el deporte, en Balaguer, I. (Dirc.) Entrenamiento Psicológico en el deporte. Principios y Aplicaciones. Valencia: Albatros Educación.

29. Feltz, D. L. (1988). Gender differences in the causal elements of selfefficacy on a high avoidance motor task. Journal of Sport and Exercise Psychology, 10, 151-166.

30. Feltz, D. L. y Petlichkoff, L. M. (1983). Perceived competence among interescholastic sport participants and dropouts. Canadian Journal of Applied Sport Science, 8, 231-235.

31. Figueiredo, A., Gonçalves, C., Cohelo e Silva, M. y Malina, R. (2009). Characsteristics of youth soccer players who dropout, persist or move up. Journal of Sports Sciences, 27 (9), 883-891.

32. Fry, M. D., McClements, J. S. y Sfton, J. M. (1981). A report on participation in the Saskatoon Hockey Association. Saskatoon, Canada: SASK SPORT.

33. Giménez, F. J., Abad, M. T., y Robles, J. (2010). El proceso de formación del jugador durante la etapa de iniciación deportiva. Apunts. Educación Física y Deportes, 99, 47-55

34. Guivernau, M. y Duda, J. L. (1994). Psychometric properties of a spanish version of the task and ego orientation in sport questionnaire (TEOSQ) and beliefs about the causes of success inventory. Revista de Psicología del Deporte, 5, 31-51.

35. González, G., Tabernero, B. y Márquez, S. (2000). Análisis de los motivos para participar en fútbol y tenis en la iniciación deportiva. Revista Motricidad, 6, 47-66.

36. González-Cutre, D., Sicilia, A., y Moreno, J.A. (2011). Un estudio cuasi-experimental de los efectos del clima motivacional tarea en las clases de educación física. Revista de Educación, 356, 677-700.

37. Gould, D.; Feltz, D.; Weiss, M. R., Petlichkoff, L-M. (1982). Participation motives in competitive youth swimmers. VWorld Congress of Sport Phychology. Ontario, Canada.

38. Granero, A. y Baena, A. (2013). Análisis preliminar exploratorio del "Sport Motivation Scale" adaptado a la Educación Física. Espiral. Cuadernos del Profesorado, 6 (12), 3-14.

39. Greghaigne, J. F. (2001). La organización del juego en fútbol. Barcelona. INDE.

40. Harter, S. (1978). Effectance motivation reconsidered: Toward a Developmental Model. Human Development, 21(1), 34-64.

41. Hirota, V. B. y De Marco, A. (2006). Identificaçao do clima motivacional em escolas publicas e particulares na aprendizagem esportiva no futebol de campo: um estudo piloto. Revista Brasileira de Educaçao Física e Esporte, 20 (5), 123-133.

42. Isorna, M., Rial, A. y Vaquero, R. (2014). Motivaciones para la práctica deportiva en escolares federados y no federados. Retos. Nuevas tendencias en Educación Física, Deporte y Recreación, 25, 80-84.

43. Junge, A., Rosch, D., Peterson, L., Baumann, T y Dvorak, J. (2002). Prevention of soccer injuries: a prospective intervention study in youth amateur players. Journal Sports Medicine, 30 (5), 652-659.

44. Kavussanu, M. y Roberts, G. C. (1996). Motivation in physical activity contexts: The relationship of perceived motivational climate to intrinsic motivation and self-efficacy. Journal of Sport and Exercise Psychology, 18, 264-280.

45. Kirkby, R. J., Kolt, G. S. y Liu, J. (1999). Participation motives of young australian and chinese gymnasts. Perceptual and Motor Skills, 88, 363373.

46. Klint, K., y Weiss, M. (1986). Dropping in and out. Participation motives of current and former youth gymnast. Canadian Journal of Applied Sport Sciences, 11, 106-114.
47. Lázaro, I., Villamarín, F., y Limonero, J.T. (1996). Motivación para participar y auto-eficacia en jóvenes jugadores de baloncesto. IV Congreso Nacional y Andaluz de la Actividad Física y el Deporte. Investigación y Aplicación. Instituto Andaluz del Deporte. Málaga.

48. Leo, F. M., Sánchez, P. A., Oliva, D., Alonso, D. y García-Calvo, T. (2012). Evolution of perceived cohesion and efficacy over the season and their relation to success expectations in soccer teams. Journal of Human Kinetics, 34, 129-138.

49. Maehr, M. L. y Nicholls, J. G. (1980). Culture and achievement motivation: A second look. En N. Warren (Ed.), Vol. 1. Studies in cross-cultural psychology (pp. 221-267). New York, NY: Academic Press.

50. Martínez, R., Bastos, A. A. y Salguero, A. (2005). Análisis factorial del cuestionario de Motivos de Participación Deportiva de Gill et al. (1983). Adaptado a una muestra de jóvenes futbolistas. $X$ Congreso Nacional $y$ Andaluz de Psicologia de la Actividad Física y el Deporte. Málaga.

51. Martínez, R., Molinero, O., Jimenez, R., Salguero, A., Tuero, C. y Márquez, S. (2008). La motivación para la práctica en la iniciación al fútbol: influencia de la edad/categoría competitiva, en el tiempo de entrenamiento y la relación con el entrenador. Apunts Educación Fisica y Deportes, 93, 46-54.

52. Medbery, R.; Gould, D. (1998). Kids tell us what about swimming. <http://www.usswim.org/coaches/funstudy.htm> [Consulta: 01/07/02]

53. Morano, M., Colella, D. y Capranica, L. (2011). Body image, perceived and actual physical abilities in normal-weight and overweight boys involved in individual and team sports. Journal of Sports Sciences, 29 (4), 355-362.

54. Morcillo, J. A. (2004). El desarrollo profesional del entrenador de fútbolbase basado en el trabajo colaborativo en un club amateur. Tesis Doctoral. Universidad de Granada. Granada.

55. McAuley, E.; y Gill, D. L. (1983). Reliability and validity of the physical self-efficacy scale in a competitive sport setting. Journal of Sport Psychology, 5, 410-418.

56. Narciso, M.; Otto, S.; Mielke, D. (1984). An analysis of reasons for athletic dropout in youth soccer programs. Soccer Journal, 29, 33-39.

57. Newton, M. y Duda, J. (1999). The interaction of motivational climate, dispositional goal, orientations, and perceived ability in predicting indices of motivation. International Journal of Sport Psychology, 30, 63-82.

58. Nicholls, J. G. (1984). The development of chievement motivation, vol. 3. Greenwich: JAI Press.

59. Nicholls, J.G. (1989). The competitive ethos and democratic education. Cambridge (MA): Harvard University Press.

60. Ommundsen, Y., Roberts, G. C., Nicolas, P. N. y Miller, W. (2005). Peer relationships in adolescent competitive soccer: Associations to perceived motivational climate, achievement goals and perfectionism. Journal of Sports Sciences, 23 (9), 977-989.

61. Ortega, M. J. y Zubiaur, M. (2003). Las orientaciones de meta y los motivos de práctica deportiva en jóvenes gimnastas de Castilla y León. $I X$ Congreso Nacional de Psicología de la Actividad Física y el Deporte. León.

62. Mesquita, I., Borges, M., Rosado, A., y De Souza, A. (2011). Handball coaches' perceptions about the value of working competences according to their coaching background. Journal of Sports Science and Medicine, 10, 193-202.

63. Moreno, J.A., Cervelló, E., Zomeño, T.E., y Marín, L.M. (2009). Predicción de las razones de disciplina en Educación Física. Acción Psicológica, 6(2), 7-15.

64. Pooley, J.C. (1980). Dropouts from sport: A case study of boy's agegroup soccer. American Association for Health, Physical Education and Recreation National Conference Congres. Boston.

65. Potgieter, R. D. y Steyn, B. J. M. (2010). Goal orientation, self-theories and reactions to success and failure in competitive sport. African Journal for Physical, Health Education,16 (4), 635-647.

66. Price M. S. y Weiss M. R. (2011). Peer leadership in sport: relationships among personal characteristics, leader behaviors, and team outcomes. 
Journal of Applied Sport Psychology, 23, 49-64.

67. Roberts, G. (1995). Motivación en el Deporte y el Ejercicio. Bilbao: Descleé de Brouwer.

68. Roberts, G. C., Kleiber, G. A. y Duda, J. L. (1981). An analysis of motivation of children's sport: The role of perceived competence in children's sport. Journal of Sport Psychology, 3, 206-216.

69. Roberts, G. C. y Treasure, D. C. (1995). Achievement goals, motivational climate and achievement strategies and behaviors in sport. International Journal of Sport Psychology, 26, 64-80.

70. Ryckman, R. M., Robbins, M. A., Thornton, B. y Cantrell, P. (1982). Development and validation of a Physical Self-Efficacy Scale. Journal of Personality and Social Psychology, 42, 891-900.

71. Sage, G. y Loudermilk, S. (1979). The female athlete and role conflict. Research Quarterly, 50, 88-96.

72. Salguero, A. (2004). Factores motivacionales que inciden en la práctica de la natación de competición y su relación con la habilidad física percibida y la deseabilidad social. Tesis Doctoral. Universidad de León. León.

73. Salguero, A., González-Boto, R., Tuero, C. y Márquez, S. (2003). La habilidad física percibida en la natación de competición. Revista Motricidad, 10, 53-69.

74. Salguero, A., González-Boto, R., Tuero, C. y Márquez, S. (2004). Relationship between perceives physical ability and sport participation motives in young competitive swimmers. Journal of Sports Medicine and Physical Fitness, 44 (3), 294-299.

75. Sans, A. y Frattarola, C. (2009). Los fundamentos del fútbol. Programa AT-3. Etapa de rendimiento. Un nuevo concepto en el que fundamentar la formación del futbolista y el entrenamiento de máximo rendimiento. Barcelona. MCsports.

76. Stratton, R.K. (1999). Coaching youth sports: Continuing to partici- pate or droppingout.http://academic.emporia.edulermlerkalethics/week13. htm [ Consulta: 24/01/01]

77. Swain, A. B. y Harwood, C. G. (1996). Antecedents of state goals in age-group swimmers: an interactionist perspective. Journal of Sport Sciences, 14 (2), 111-24.

78. Ullrich-French, S. y Smith, A. (2009). Social and motivational predictors of continued youth sport participation. Psychology of Sport and Exercise 10, 87-95.

79. Vickers, B., y Schoenstedt, L. (2011). Coaching development: Methods for youth sport introduction. Strategies: A journal for Physical and Sport Educators, 1, 14-19.

80. Villamarín, F., Mauri, C. y Sanz, A. (1998). Competencia percibida y motivación durante la iniciación a la práctica del tenis. Revista de Psicología del Deporte, 13, 41-56.

81. White, S. A. y Duda, J. L. (1994). The relationship of gender, level of sport involvement, and participation motivation to task and ego orientation. International Journal of Sport Psychology, 25, 4-18.

82. Weigand, D. A. y Broadhurst, C. J. (1988). The relationship among perceived competence, intrinsic motivation and control perceptions in youth soccer. International Journal of Sport Psychology, 29, 324-338.

83. Weinberg, R. S. y Gould, D. (1996). Fundamentos de psicología del deporte y el ejercicio físico. Barcelona: Editorial Ariel.

84. Weiss, M. R.; Bredemeier, B. J.; y Shewchuk, R. M. (1985). An intrinsic/extrinsic motivation scale for the youth sport setting: A confirmatory factor analysis. Journal of Sport Psychology, 7, 75-91.

85. Wood, G. y Wilson, M. R. (2012). Quiet-eye training, perceived control and performing under pressure. Psychology of Sport and Exercise, 13, 721-728. 